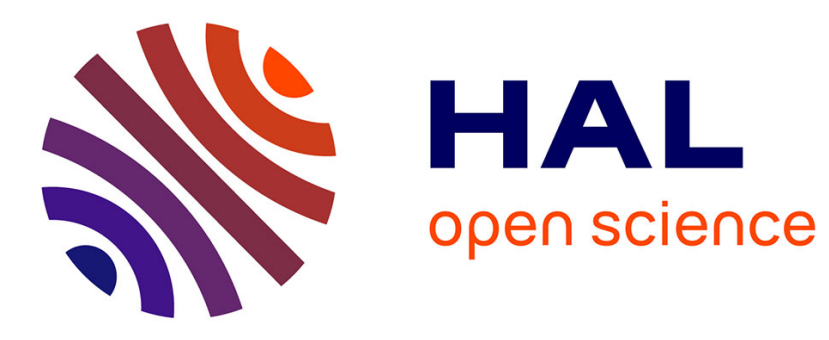

\title{
Update Operators for Inconsistent Query Answering: A New Point of View
}

Ricardo Rodriguez, Madalina Croitoru

\section{To cite this version:}

Ricardo Rodriguez, Madalina Croitoru. Update Operators for Inconsistent Query Answering: A New Point of View. SUM: Scalable Uncertainty Management, Sep 2014, Oxford, United Kingdom. pp.100105, 10.1007/978-3-319-11508-5_9. lirmm-01088098

\section{HAL Id: lirmm-01088098 https://hal-lirmm.ccsd.cnrs.fr/lirmm-01088098}

Submitted on 27 Nov 2014

HAL is a multi-disciplinary open access archive for the deposit and dissemination of scientific research documents, whether they are published or not. The documents may come from teaching and research institutions in France or abroad, or from public or private research centers.
L'archive ouverte pluridisciplinaire HAL, est destinée au dépôt et à la diffusion de documents scientifiques de niveau recherche, publiés ou non, émanant des établissements d'enseignement et de recherche français ou étrangers, des laboratoires publics ou privés. 


\title{
Update Operators for Inconsistent Query Answering: A New Point of View
}

\author{
Madalina Croitoru ${ }^{1}$, Ricardo Oscar Rodriguez ${ }^{2}$ \\ 1 University of Montpellier 2, France \\ croitoru@lirmm.fr \\ ${ }^{2}$ University of Buenos Aires, Argentina \\ ricardo@dc.uba.ar
}

\begin{abstract}
This paper investigates the definition of belief revision operators that correspond to the IAR and ICR inconsistency tolerant semantics in the Ontology Based Data Access (OBDA) setting. By doing this equivalence our long term goal is to provide an axiomatic characterisation of the above mentioned semantics.
\end{abstract}

\section{Introduction and Motivation}

We position ourselves in the Ontology Based Data Access (OBDA) setting where a query is being asked over a set of knowledge bases defined over a common ontology. When the union of knowledge bases along with the ontology is inconsistent, several semantics have been defined $[5,11]$ which are tolerant to inconsistency. They all rely on computing repairs, i.e. maximal (in terms of set inclusion) subsets of the knowledge bases. The inconsistency tolerant semantics (Intersection of All Repairs: IAR, All Repairs: AR, Intersection of Closed Repairs: ICR) have been studied [5,11], from a productivity point of view and a complexity point of view.

In this paper we take a new point of view. Our long term aim is to define axiomatic characterisations of two such semantics (IAR and ICR). We argue that such characterisation can provide an alternative way of comparing the semantics and can provide new insights into their properties. Furthermore such axiomatisation can be used when proposing a generalisation of inconsistency tolerant semantics.

In order to provide the axiomatic characterisation we define belief revision operators that correspond to IAR and ICR. The belief revision operator corresponding to the AR semantics is left for future work. Please note that while a lot of work has been done in belief revision and OBDA, none of the approaches deal with the final goal of their axiomatic characterisations. The paper is structures as follows: Section 2 introduces the rule based OBDA language used in the paper, Section 3 defines the revision operators that correspond to ICR and IAR. Based on the axiomatic characterisation of the belief operators we can provide the axiomatic characterisation of IAR and ICR. Finally, Section 4 concludes the paper.

\section{Rule Based Knowledge Representation}

There are two major approaches in the literature used to represent an ontology for the OBDA problem and namely Description Logics (such as $\mathcal{E} \mathcal{L}([3])$ and DL-Lite [7] fami- 
lies) and rule based languages. The most notable rule based language is the Datalog ${ }^{+}[6]$ language, a generalization of Datalog that allows for existentially quantified variables in the head of the rules. Despite Datalog ${ }^{+}$undecidability when answering conjunctive queries, there exist decidable fragments of Datalog ${ }^{+}$are studied in the literature [4]. These fragments generalize the above mentioned Description Logics families.

In this paper we represent the ontology via rules. We consider a (potentially inconsistent) knowledge base composed of a set $\mathcal{F}$ of facts corresponding to existentially closed conjunctions of atoms, which can contain $n$-ary predicates; a set of negative constraints $\mathcal{N}$ which represent the negation of a fact and an ontology composed of a set of rules $\mathcal{R}$ that represent general implicit knowledge that can introduce new variables in their head (conclusion).

A rule is applicable to set of facts $\mathcal{F}$ if and only if the set entails the hypothesis of the rule. If rule $R$ is applicable to the set $\mathcal{F}$, the application of $R$ on $\mathcal{F}$ produces a new set of facts obtained from the initial set with additional information from the rule conclusion. We then say that the new set is an immediate derivation of $\mathcal{F}$ by $R$ denoted by $R(\mathcal{F})$. Let $\mathcal{F}$ be a set of facts and let $\mathcal{R}$ be a set of rules. A set $\mathcal{F}_{n}$ is called an $\mathcal{R}$ derivation of $\mathcal{F}$ if there is a sequence of sets (derivation sequence) $\left(\mathcal{F}_{0}, \mathcal{F}_{1}, \ldots, \mathcal{F}_{n}\right)$ such that: (i) $\mathcal{F}_{0} \subseteq \mathcal{F}$, (ii) $\mathcal{F}_{0}$ is $\mathcal{R}$-consistent, (iii) for every $i \in\{1, \ldots, n-1\}$, it holds that $\mathcal{F}_{i}$ is an immediate derivation of $\mathcal{F}_{i-1}$.

Given a set $\left\{F_{0}, \ldots, F_{k}\right\}$ and a set of rules $\mathcal{R}$, the closure of $\left\{F_{0}, \ldots, F_{k}\right\}$ with respect to $\mathcal{R}$, denoted $\mathrm{Cl}_{\mathcal{R}}\left(\left\{F_{0}, \ldots, F_{k}\right\}\right)$, is defined as the smallest set (with respect to $\subseteq$ ) which contains $\left\{F_{0}, \ldots, F_{k}\right\}$, and is closed for $\mathcal{R}$-derivation (that is, for every $\mathcal{R}$-derivation $F_{n}$ of $\left\{F_{0}, \ldots, F_{k}\right\}$, we have $\left.F_{n} \subseteq \mathrm{Cl}_{\mathcal{R}}\left(\left\{F_{0}, \ldots, F_{k}\right\}\right)\right)$. Finally, we say that a set $\mathcal{F}$ and a set of rules $\mathcal{R}$ entail a fact $G$ (and we write $\mathcal{F}, \mathcal{R}=G$ ) iff the closure of the facts by all the rules entails $G$ (i.e. if $\mathrm{Cl}_{\mathcal{R}}(\mathcal{F}) \models G$ ). Given a set of facts $\left\{F_{1}, \ldots, F_{k}\right\}$, and a set of rules $\mathcal{R}$, the set of facts is called $\mathcal{R}$-inconsistent if and only if there exists a constraint $N=\neg F$ such that $\mathrm{Cl}_{\mathcal{R}}\left(\left\{F_{1}, \ldots, F_{k}\right\}\right) \models F$. A set of facts is said to be $\mathcal{R}$-consistent iff it is not $\mathcal{R}$-inconsistent. A knowledge base $\mathcal{K}=(\mathcal{F}, \mathcal{R}, \mathcal{N})$ is said to be consistent if and only if $\mathcal{F}$ is $\mathcal{R}$-consistent. A knowledge base is inconsistent if and only if it is not consistent.

Several semantics have been proposed to handle consistency based in the concept of data repairs [5, 11]. Once the repairs are computed, various strategies can be adapted to answer a query. We can consider querying all repairs (AR-semantics), the intersection of all repairs (IAR-semantics) or the intersection of closed repairs (ICR-semantics).

Definition 1. $[5,11]$ Let $\mathcal{K}=(\mathcal{F}, \mathcal{R}, \mathcal{N})$ be a knowledge base and let $\alpha$ be a query. Then $\alpha$ is $\boldsymbol{A R}$-entailed from $\mathcal{K}$, written $\mathcal{K} \models_{A R} \alpha$ iff for every repair $A^{\prime} \in \mathcal{R}$ epair $(\mathcal{K})$, it holds that $\mathrm{Cl}_{\mathcal{R}}\left(A^{\prime}\right) \models \alpha$.

Definition 2. $[5,11]$ Let $\mathcal{K}=(\mathcal{F}, \mathcal{R}, \mathcal{N})$ be a knowledge base and let $\alpha$ be a query. Then $\alpha$ is IAR-entailed from $\mathcal{K}$, written $\mathcal{K} \models_{I A R} \alpha$ iff $\mathrm{Cl}_{\mathcal{R}}\left(\bigcap_{A^{\prime} \in \operatorname{Repair}(\mathcal{K})}\right) \models \alpha$.

Definition 3. $[5,11]$ Let $\mathcal{K}=(\mathcal{F}, \mathcal{R}, \mathcal{N})$ be a knowledge base and let $\alpha$ be a query. Then $\alpha$ is ICR-entailed from $\mathcal{K}$, written $\mathcal{K} \models_{I C R} \alpha$ iff $\bigcap_{A^{\prime} \in \mathcal{R} \text { epair }(\mathcal{K})} \mathrm{Cl}_{\mathcal{R}}\left(A^{\prime}\right) \models \alpha$. 


\section{Belief Revision Operators}

In the context of Belief Revision, the problem of making an inconsistent belief base consistent was solved by Hanson in [10] who proposed a new operation called Consolidation. An inconsistent belief base can be consolidated by removing some of its elements. The consolidation of a belief base $B$ is denoted $B$ !. A plausible way to perform consolidation is to contract by falsum (contradiction), i.e. $B !=B \div \perp$. In order to express this notion more precisely, we need to introduce the concept of remainder set proposed by Alchourrón and Makinson in [2]. For any belief base $B$ and any formula $\alpha$, the remainder set of $B$ by $\alpha, B \perp \alpha$, is the set of maximal subsets of $B$ that do not imply $\alpha$. In other words, in our language:

Definition 4. (Updated AM81 [2]) Let $(\mathcal{F}, \mathcal{R}, \mathcal{N})$ a knowledge base and $\alpha$ a fact in $\mathcal{F}$. The set $\mathcal{F} \perp \alpha$ (read " $\mathcal{F}$ less $\alpha$ ") is the set such that $A$ belongs to $\mathcal{F} \perp \alpha$ if and only if $A \subseteq \mathcal{F}, \mathrm{Cl}_{\mathcal{R}}(A) \not \forall \alpha$ and there is no set $A^{\prime}$ such that $A \subset A^{\prime} \subseteq \mathcal{F}$ and $\mathrm{Cl}_{\mathcal{R}}\left(A^{\prime}\right) \forall \neq \alpha$.

Definition 5. A selection function is a function $\gamma$ such that for every set $\mathcal{F}$ of formulae and any fact $\alpha$ it holds: $\gamma(\mathcal{F} \perp \alpha)$ is a non-empty subset of $\mathcal{F} \perp \alpha$ if this set is nonempty, and, on the contrary, $\gamma(\mathcal{F} \perp \alpha)=\{\mathcal{F}\}$.

Definition 6. (Updated AGM85 [1]) Let $\mathcal{F}$ be a set offacts in a knowledge base $\mathcal{K}$. Let $\mathcal{F} \perp \alpha$ and $\gamma$ be the set of all maximal subsets of $\mathcal{F}$ that do not imply $\alpha$ and a selection function, respectively. The partial meet contraction on $\mathcal{F}$ that is generated by $\gamma$ is the operation $\sim_{\gamma}$ such that for all facts $\alpha:$

$$
\mathcal{F} \sim_{\gamma} \alpha=\cap \gamma(\mathcal{F} \perp \alpha)
$$

Two limiting cases have been very well studied: when $\gamma$ gives back either only one element of $\mathcal{F} \perp \alpha$ or all members of $\mathcal{F} \perp \alpha$. In the first case, we are talking about Maxichoice contraction and in the second it is called Full meet contraction.

There are other special and interesting cases when the selection function is based on a relation (that may be thought of a preference relation).

Definition 7. A selection function $\gamma$ for a belief base $\mathcal{F}$ in a knowledge base $\mathcal{K}$, and the contraction operator based on it, are

1. relational if and only if there is a binary relation $\sqsubseteq$ such that for all fact $\alpha$, if $\mathcal{F} \perp \alpha$ is non-empty, then

$$
\gamma(\mathcal{F} \perp \alpha)=\{A \in \mathcal{F} \perp \alpha \mid C \sqsubseteq A \text { for all } C \in \mathcal{F} \perp \alpha\}
$$

2. transitively relational if and only if there is such a relation that is transitive.

Based on partial meet contraction, one can define a partial meet consolidation as $\mathcal{F} \sim_{\gamma} \perp$ which is the intersection of the "most preferred" maximal consistent subsets of $\mathcal{F}$, i.e. $\mathcal{F}$ ! $=\mathcal{F} \sim_{\gamma} \perp=\cap \gamma(\mathcal{F} \perp \perp)$ where $\perp$ denotes logical contradiction.

Partial meet consolidation has been axiomatically characterized as follows: 
Theorem 1. ( Updated [9]) An operation is a partial meet consolidation if and only if for all sets $\mathcal{F}$ of facts the following are satisfied:

Consistency: $\mathcal{F} !$ is $\mathcal{R}$-consistent.

Inclusion: $\mathcal{F} ! \subseteq \mathcal{F}$.

Relevance: If $\alpha \in \mathcal{F} \backslash \mathcal{F}$ !, then there is some $\mathcal{F}^{\prime}$ with $\mathcal{F}$ ! $\subseteq \mathcal{F}^{\prime} \subseteq \mathcal{F}$, such that $\mathcal{F}^{\prime}$ is $\mathcal{R}$-consistent and $\mathcal{F}^{\prime} \cup\{\alpha\}$ is $\mathcal{R}$-inconsistent.

In addition, it is a full meet consolidation if and only if it also satisfies:

Core identity: $\beta \in \mathcal{F}$ ! if and only if $\beta \in \mathcal{F}$ and there is no $\mathcal{F}^{\prime} \subseteq \mathcal{F}$ such that $\mathcal{F}^{\prime}$ is $\mathcal{R}$-consistent but $\mathcal{F}^{\prime} \cup\{\beta\}$ is $\mathcal{R}$-inconsistent.

On the other hand, an operator is a maxi-choice consolidation if and only if it satisfies the postulates consistency, inclusion, and:

Fullness: If $\beta \in \mathcal{F}$ and $\beta \notin \mathcal{F}$ ! then $\mathcal{F} ! \cup\{\beta\}$ is $\mathcal{R}$-inconsistent.

There are five ways to characterise a belief revision function: axiomatic, using remainder sets, using kernel sets, epistemic entrenchment and spheres system. In the following we will define the operators corresponding to the inconsistency tolerant semantics above using its axiomatic characterisation.

\subsection{Consolidation operators for existing semantics}

We want to define an operator of consolidation that given a inconsistent knowledge base $\mathcal{K}=(\mathcal{F}, \mathcal{R}, \mathcal{N})$, returns a new consistent knowledge base $\mathcal{K} !=(\mathcal{F} !, \mathcal{R}, \mathcal{N})$.

As a first step, we consider the maximal consistent subsets (repairs or remainders) of $\mathcal{F}$ denoted $\mathcal{K} \perp \perp$. More precisely:

$$
\mathcal{K} \perp \perp=\{\mathcal{M} \mid \mathcal{M} \subseteq \mathcal{F},(\mathcal{M}, \mathcal{R}, \mathcal{N}) \text { maximal consistent set }\}
$$

We are now able to define an operator of consolidation as $\mathcal{K} !=O p(C h(\mathcal{K} \perp \perp))$ where $C h$ is a choice function and $O p$ an intersection operator (or an any operator defined over the results of choice). It is easy to check the following:

\section{If Ch returns all elements and $O p$ is the intersection of sets then we get the IAR semantics.}

Note that in this case we are talking about a full meet consolidation and it allows us to give immediately an axiomatic characterization of IAR semantics.

In order to obtain the equivalent of ICR semantics we need to work on sets closed by $\mathrm{Cl}_{\mathcal{R}}$. First, we have to introduce some properties.

Observation 1 If $\mathcal{M} \in \mathcal{K} \perp \perp$ then $\mathrm{Cl}_{\mathcal{R}}(\mathcal{M}) \cap \mathcal{F} \subseteq \mathcal{M}$. Hence, if $\mathcal{F}$ is closed by $\mathcal{R}$-derivation (i.e. $\mathcal{F}=\mathrm{Cl}_{\mathcal{R}}(\mathcal{F})$ ) then $\mathcal{M}$ is as well. 
We now define the remainders or repairs sets closed by $\mathrm{Cl}_{\mathcal{R}}$ in the following way:

$$
\mathcal{K} \perp_{\mathrm{Cl}_{\mathcal{R}}} \perp=\left\{\mathcal{M} \mid \mathcal{M} \subseteq C l_{\mathcal{R}}(\mathcal{F}),(\mathcal{M}, \mathcal{R}, \mathcal{N}) \text { maximal consistent set }\right\}
$$

We are now able to define a new family of consolidation operators using these remainders. This kind of operators defined over closed sets by $\mathcal{R}$-derivations will be called closure partial meet consolidation operators and denoted by $\dagger$. We also cover the ICR semantics as follows:

2. If $C h$ returns all elements and $O p$ is the intersection of sets over $\{\mathcal{M} \mid \mathcal{M} \in$ $\left.\mathcal{K} \perp_{\mathrm{Cl}_{\mathcal{R}}} \perp\right\}$ then we get the ICR semantics.

Note that we describe a full meet consolidation but over remainders closed by $\mathcal{R}$ derivations. In order to give an axiomatization of this kind of consolidations, we need to included an axiom which mirrors this fact. This will be done in the next section.

\subsection{Axiom Compliance}

In this section we go one step further in the definition of consolidation operators in relationship with ICR semantics through a set of postulates. In order to give logical properties of that kind of consolidation operators, we first rephrase Hansson's postulates within our framework. Let $\mathcal{K}=(\mathcal{F}, \mathcal{R}, \mathcal{N})$ be a knowledge base, the original postulates can be rewritten in following way:

Closure: $\mathcal{F} \dagger=\mathrm{Cl}_{\mathcal{R}}(\mathcal{F} \dagger)$.

Consistency: $\mathcal{K} \dagger=(\mathcal{F} \dagger, \mathcal{R}, \mathcal{N})$ is consistent.

Inclusion: $\mathcal{K} \dagger=(\mathcal{F} \dagger, \mathcal{R}, \mathcal{N}) \sqsubseteq \mathcal{K}=(\mathcal{F}, \mathcal{R}, \mathcal{N})$.

Core identity: If $f \in \mathcal{F} \dagger$ if and only if $f \in \mathrm{Cl}_{\mathcal{R}}(\mathcal{F})$ and $\nexists \mathcal{X} \subseteq \mathrm{Cl}_{\mathcal{R}}(\mathcal{F})$, such that $\mathcal{X}$ is $\mathcal{R}$-consistent and $\mathcal{X} \cup f$ does not.

Theorem 2. An operator $\dagger$ is an operation of closure full meet consolidation if and only if it satisfies Closure, Consistency, Inclusion and Core identity.

Proof Checking that operations of closure full meet consolidation satisfy postulates: Closure, Consistency and Inclusion follow directly from definition and Observation 1. To see that closure full meet consolidation satisfies Core identity, let $f \in \mathrm{Cl}_{\mathcal{R}}(\mathcal{F})$. Then $f \notin \mathcal{F} \dagger$ if and only if there is some $\mathcal{X} \in \mathcal{K} \perp_{\mathrm{Cl}_{\mathcal{R}}} \perp$ such that $f \notin \mathcal{X}$. Since $\mathcal{X}$ is a maximal $\mathcal{R}$-consistent set it follows that $\mathcal{X} \cup f$ does not. Hence, $\mathcal{X}$ satisfies the required properties by Core Identity.

On the contrary direction, let $\dagger$ be an operator that satisfies the four postulates mentioned in the theorem. We need to show that $\mathcal{F} \dagger=\bigcap \mathcal{K} \perp_{\mathrm{Cl}_{\mathcal{R}}} \perp$. We assume again, that $f \in \mathrm{Cl}_{\mathcal{R}}(\mathcal{F})$. Suppose that $f \notin \bigcap \mathcal{K} \perp_{\mathrm{Cl}_{\mathcal{R}}} \perp$ then there is $\mathcal{X} \in \mathcal{K} \perp_{\mathrm{Cl}_{\mathcal{R}}} \perp$ such that $f \notin \mathcal{X}$. Since $\mathcal{X}$ is maximal $\mathcal{R}$-consistent then $\mathcal{X} \cup\{f\}$ does not. From that and Core Identity we conclude that $f \notin \mathcal{F} \dagger$. On the other hand, since $\dagger$ satisfies Inclusion we can conclude that $\mathcal{F} \dagger \subseteq \mathrm{Cl}_{\mathcal{R}}(\mathcal{F})$. In addition, because $\dagger$ satisfies Closure and Consistency, we may assume that there exists $f \notin \mathcal{F} \dagger$. It follows by Core Identity 
that for each $f \notin \mathcal{F} \dagger$ there exists $\mathcal{X} \subset \mathrm{Cl}_{\mathcal{R}}(\mathcal{F})$ such that $\mathcal{X}$ is $\mathcal{R}$-consistent and $\mathcal{X} \cup f$ does not. In the usual way we are able to extend $\mathcal{X}$ to a maximal $\mathcal{R}$-consistent set $\mathcal{X}^{\prime}$ such that $f \notin \mathcal{X}^{\prime}$. Since $\mathcal{X} \subset \mathrm{Cl}_{\mathcal{R}}(\mathcal{F})$ then $\mathcal{X}^{\prime} \subset \mathrm{Cl}_{\mathcal{R}}(\mathcal{F})$ and $\mathcal{X}^{\prime} \in \mathcal{K} \perp_{\mathrm{Cl}_{\mathcal{R}}} \perp$. From the last, we may conclude that $f \notin \mathcal{K} \perp_{\mathrm{Cl}_{\mathcal{R}}} \perp$. This finishes the proof.

\section{Conclusion and future work}

In this paper we showed how to get first results towards an axiomatic characterisation of the IAR and ICR semantics in the OBDA setting. We did this by covering the semantics using known consolidation operators from belief revision. The AR semantics poses some problems. Indeed, to cover the AR semantics let $\left\{C h_{i}\right\}$ with $1 \leq i \leq \#(\mathcal{K} \perp \perp)$ be a family of choice functions such that each $C h_{i}$ returns only one different element of $\mathcal{K} \perp_{\mathrm{Cl}_{\mathcal{R}}} \perp$, i.e. we enumerate the elements of $\mathcal{K} \perp_{\mathrm{Cl}_{\mathcal{R}}} \perp$ and each $C h_{i}$ gives back only one of it. If we consider the family of maxichoice consolidation defined by $\left\{C h_{i}\right\}$ and we take the intersection among the classical consequence closure of each $\mathcal{K}_{i}$ ! then we get the AR semantics. In this case, we are considering a family of maxichoice consolidations which have also a well known axiomatic characterization. But it is not clear how to axiomatize the combination proposed here. This will be left for future research. Note that by using the equivalence showed in [8] we also obtained here an axiomatic characterisation of some argumentation semantics.

ACKNOWLEDGEMENTS: The first author was funded by the ANR ASPIQ project.

\section{References}

1. Alchourrón, C., Gärdenfors, P., Makinson, D.: On the logic of theory change: partial meet contraction and revision function. Journal Symbolic Logic 50, 510-530 (1985)

2. Alchourrón, C., Makinson, D.: Deontic Logic:Introductory and Systematic Readings, chap. Hierarchies of regulations and their logic, pp. 125-148. Reidel Publishing Company, Dordrecht, r. hilpinen edn. (1981)

3. Baader, F., Brandt, S., Lutz, C.: Pushing the el envelope. In: Proc. of IJCAI 2005 (2005)

4. Baget, J.F., Mugnier, M.L., Rudolph, S., Thomazo, M.: Walking the complexity lines for generalized guarded existential rules. In: Proceedings of the 22nd International Joint Conference on Artificial Intelligence, (IJCAI'11). pp. 712-717 (2011)

5. Bienvenu, M.: On the complexity of consistent query answering in the presence of simple ontologies. In: Proc of AAAI (2012)

6. Calì, A., Gottlob, G., Lukasiewicz, T.: A general datalog-based framework for tractable query answering over ontologies. In: Proceedings of the Twenty-Eigth ACM SIGMOD-SIGACTSIGART Symposium on Principles of Database Systems. pp. 77-86. ACM (2009)

7. Calvanese, D., De Giacomo, G., Lembo, D., Lenzerini, M., Rosati, R.: Tractable reasoning and efficient query answering in description logics: The dl-lite family. J. Autom. Reasoning 39(3), 385-429 (2007)

8. Croitoru, M., Vesic, S.: What can argumentation do for inconsistent ontology query answering? In: Proc of SUM. pp. 15-29 (2013)

9. Hansson, S.: Belief Base Dynamics. Ph.D. thesis, Uppsala (1991)

10. Hansson, S.: Semi-revision. Journal of Applied Non-Classical Logics 7(1-2), 151-175 (1997)

11. Lembo, D., Lenzerini, M., Rosati, R., Ruzzi, M., Savo, D.F.: Inconsistency-tolerant semantics for description logics. In: Proc. of RR. pp. 103-117 (2010) 